\title{
Relationship between biomarkers of muscle damage and redox status in response to a weightlifting training session: effect of time-of-day
}

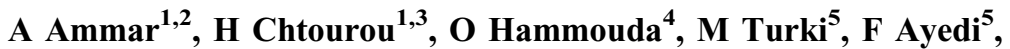 \\ C Kallel $^{6}$, O AbdelKarim ${ }^{2}$, A Hoekelmann ${ }^{2}$, N Souissi ${ }^{7}$ \\ ${ }^{1}$ Research Unit: Education, Motricity, Sport and Health, High Institute of Sport and Physical Education \\ of Sfax, Sfax University, Sfax, Tunisia \\ ${ }^{2}$ Institute of Sport Science, Otto-von-Guericke-University Magdeburg, Magdeburg, Germany \\ ${ }^{3}$ High Institute of Sport and Physical Education of Sfax, Sfax University, Sfax, Tunisia \\ ${ }^{4}$ Research Center on Sport and Movement (CeRSM, EA 2931), Sciences and Techniques of the Physical and \\ Sporting Activities (UFR STAPS), University of Paris Ouest Nanterre La Defense, Nanterre, France \\ ${ }^{5}$ Laboratory of Biochemistry, CHU Habib Bourguiba, Sfax University, Sfax, Tunisia \\ ${ }^{6}$ Laboratory of Hematology, CHU Habib Bourguiba, Sfax University, Sfax, Tunisia \\ ${ }^{7}$ National Sport Observatory, Tunis, Tunisia
}

Received: October 13, 2015

Accepted: May 17, 2016

The aims of the present study were to: (1) investigate the effect of a weightlifting training session and time-of-day (TOD) upon biological parameters (i.e., oral temperature, hematological, C-reactive protein (CRP), and oxidative stress) and (2) assess their possible link with muscle damage responses. Nine weightlifters $(21 \pm 0.5$ years $)$ performed, in a randomized order, three Olympic-Weightlifting sessions (i.e., at 08:00, 14:00, and 18:00). Blood samples were collected at rest, $3 \mathrm{~min}$ and $48 \mathrm{~h}$ after each training session. Between pre- and post-training session, ANOVA showed significant increases in oxidative stress markers at the three TODs $(p<0.01)$ and significant increases for creatine kinase $(\mathrm{CK})$ and lactate dehydrogenase $(\mathrm{LDH})$ only at 08:00 and 18:00 $(p<0.05)$. At rest, the results showed a significant diurnal variation for the majority of the selected parameters except for malondialdehyde (MDA), total bilirubin, and CRP with higher values observed at 18:00 $(p<0.05)$. After the training session, given the higher rate of increase during the morning session, these diurnal variations persisted for temperature and WBC $(p<$ 0.01 ) and were suppressed for $\mathrm{CK}, \mathrm{LDH}$, uric acid (UA), catalase, and glutathione peroxidase. The main significant correlations $(p<0.001)$ were observed between: (1) CK and MDA $(r=0.6)$ and CK and UA $(r=0.66$ and $r=0.82)$ during the morning and evening training sessions; (2) CK and CRP only during the morning session ( $r=0.5)$; and (3) CRP and WBC during the three training sessions ( $r=0.8)$. In conclusion, the present findings: (1) confirm that the muscle damage responses could be induced by a high level of oxidative stress and (2) suggest to avoid scheduling training sessions in the morning given the higher muscle damage, inflammatory, and oxidative responses at this TOD.

Keywords: lipid peroxidation, creatine kinase, lactate dehydrogenase, strength exercises, diurnal variation

\section{Introduction}

Creatine kinase (CK) and lactate dehydrogenase (LDH) levels have previously been monitored in untrained and trained subjects, who underwent resistance exercise sessions, to assess muscle damage responses $(10,29)$. These parameters as well as the white blood cells (WBCs)

Corresponding author: Achraf Ammar

High Institute of Sport and Physical Education, Sfax University

Route de l'Aéroport, Km 3,5, BP 384, Sfax 3000, Tunisia

Phone: +216 20420 326; Fax: +216 74278 502; E-mail: ammar.achraf@ymail.com 
and their subpopulations have also been identified as indicators of muscle fatigue and overtraining $(35,45)$. It has been shown that rugby sevens match $(41)$ or high-intensity maximal tasks such as intermittent running, sprinting, jumping, resistance exercises $(33,45)$, or Wingate test (16) resulted in transient leukocytosis and increase in biochemical markers of muscular damage for up to 10 days after exercise (10). Particularly, after a single weightlifting session (3) or 1 week of intensive weightlifting training $(25,33)$, a profound increase (i.e., up to 2 days and 1 week, respectively) of the above-mentioned parameters have been reported. On the other hand, resting $\mathrm{WBC}, \mathrm{CK}$, and $\mathrm{LDH}$ have been shown to be affected by circadian rhythms in weightlifters (3) and soccer players (16) with acrophases generally observed at 17:00 close to the peak values of the oral temperature (36). In addition, a transient leukocytosis and increase in muscle damage have been reported after high-intensity exercise (e.g., repeated sprint and Wingate test) with higher post-exercise values observed at 17:00 compared to 07:00 (16-18). However, after a weightlifting training session, an alteration of the diurnal variation of $\mathrm{WBC}$, CK, and LDH has been reported (3). Despite the important number of studies in this field, the exact mechanisms underlying these fluctuations are, yet, not well known $(17,18)$.

It has been shown that the diurnal variation of $\mathrm{CK}, \mathrm{WBC}$, and neutrophils (NE) variations was significantly correlated to the changes in malondialdehyde (MDA) levels $(1,25)$. Indeed, exercise-induced lipid peroxidation - as a part of normal metabolism that may cause myocellular damage (37) - could be in the origin of hematological and muscle damage circadian rhythm and could even alter the whole circadian system (11). In addition, it has also been suggested that increases in total bilirubin (TBIL) and uric acid (UA) levels are thought to reflect the imbalances in protein metabolic homeostasis and increased protein catabolism associated with tissue damage (i.e., CK and LDH increases) (32). Moreover, the previous studies have confirmed that there is a causal link between the diurnal variation of resting $\mathrm{WBC}, \mathrm{CK}$, and LDH and the circadian rhythm of core temperature in soccer players (16). From the evidence of similarity in the time of peak between temperature, muscle damage, and MDA levels (20), Kanabrocki et al. (22) demonstrated a correlation between increased oxidative damage and increased rate of anabolic-catabolic events (e.g., oral temperature). Furthermore, a causal relationship has been reported between inflammatory responses and generalized muscle damage after strenuous exercise (42).

In view of the above considerations, it seems that the origin of hematological and muscle damage alterations in response to physical exercises or to different time-of-day (TOD) has not been clarified yet. Divergences between studies could be due to the participants' training levels, temporal specificity of training, and the lack of studies which investigated all the parameters under the same condition. Therefore, the aims of the present study were to: (1) investigate the diurnal variation of oral temperature, hematological and inflammatory parameters, and oxidative stress markers before and after weightlifting training sessions and (2) assess the possible relationship of these parameters with the circadian rhythm of muscle damage.

We hypothesize that intense weightlifting exercises could exert a strong enough influence to alter the circadian profile of the different parameters studied and that a causal relationship could be established between markers of muscle damage and oxidative stress responses.

\section{Materials and Methods}

\section{Experimental design}

For the purpose of the present study, the participants randomly performed snatch, clean and jerk, and the squat exercise during weightlifting training sessions scheduled at different TODs 


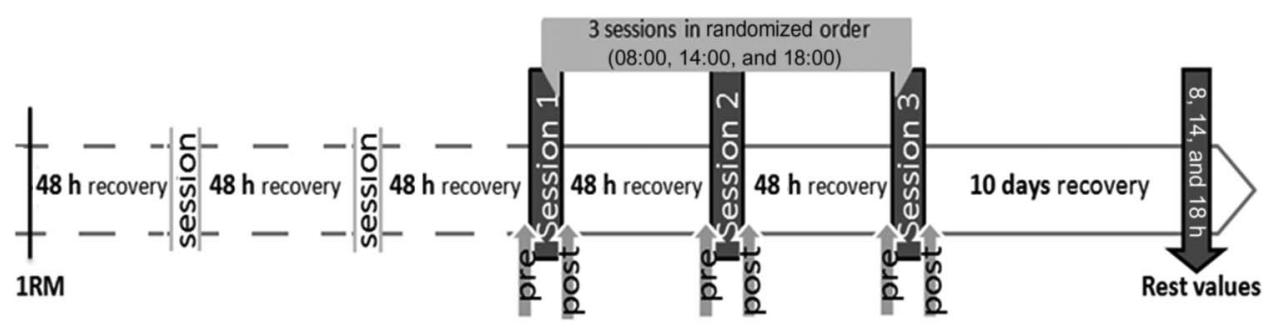

Fig. 1. Experimental design.

$N B$ : the pre-session values correspond to the values of $48 \mathrm{~h}$ of recovery

(i.e., in the morning between 08:00 and 09:45, in the afternoon between 14:00 and 15:45, and in the evening between 18:00 and 19:45) with at least $48 \mathrm{~h}$ of recovery period after each session (Fig. 1). Olympic exercises and training hours were chosen according to the peak and the minimum of circadian rhythm of anaerobic performances and with regard to the findings reported in the literature $(2,3,16)$.

\section{Participants}

Nine male elite weightlifters [age: $21 \pm 0.5$ years, body mass: $80 \pm 9.5 \mathrm{~kg}$, and height: $175 \pm$ $8.1 \mathrm{~cm}($ mean $\pm \mathrm{SD})]$ were volunteered to participate in the present study. They were recruited on the basis of: (1) at least five sessions of training per week (between 15:30 and 17:30) with 90-120 min per session and (2) more than three years of experience in Olympic weightlifting. After receiving a thorough explanation of the possible risks and discomforts associated with the experimental procedures, the participants provided a written informed consent to take part in the experiment. The study was conducted according to the Declaration of Helsinki. The protocol and the consent form were fully approved by the review board "Local committee of the Laboratory of Biochemistry, CHU Habib Bourguiba, Sfax, Tunisia" before the commencement of the assessments. Answers to the Dosseville et al. (13), the questionnaire categorized the subjects as "moderately morning type" $(n=1)$ or "neither type" $(n=8)$.

\section{Procedures}

One week before the start of the experimental period, the heaviest weight lifted in a single repetition (1-RM) was assessed for each participant in each movement. After an ascending warm-up (i.e., first set with $8-10$ repetitions at 50\%, second set with $3-5$ repetitions at $75 \%$, and third set with 1-3 repetitions at $90 \%$ of the athlete's estimated maximum), 1-RM was determinated in three trials with 5 min of recovery time in-between (31). As suggested by Kraemer and Fry (24), they emphasize that on-going encouragement and communication with the athletes during this testing is crucial to obtain the best performance. In addition, the estimated 1-RM was verified in the following 2 days. Then, three training sessions in a randomized order with a recovery period of at least $48 \mathrm{~h}$ were performed in the: morning (08:00-09:45), afternoon (14:00-15:45), and evening (18:00-19:45). Upon arrival for their first test session, each participant's body mass (Tanita, Tokyo, Japan) and height were recorded. Moreover, before and after each training session, oral temperature was measured with a calibrated digital clinical thermometer (Omron, Paris, France; accuracy: $0.05{ }^{\circ} \mathrm{C}$ ) inserted sublingually for at least $3 \mathrm{~min}$ the subjects being seated in a resting position for at least $15 \mathrm{~min}$. Before the morning test session, participants were allowed to drink only 
one glass of water to avoid the effects of post-prandial thermogenesis (9). Moreover, as recommended by Bougard et al. (9), they were requested to ingest a standardized meal at least $4 \mathrm{~h}$ before the afternoon and evening test sessions and they were not allowed to eat anything else until the end of the testing sessions.

\section{Training session}

Each training session includes three Olympic-Weightlifting exercises: snatch, clean and jerk, and the squat $(2,3,26)$ with five sets for each exercise (i.e., two sets at $85 \%$ of 1 -RM with three repetitions per set and three sets at $90 \%$ of $1-\mathrm{RM}$ with two repetitions per set) with a passive recovery period of $5 \mathrm{~min}$ in-between (6). Each session was preceded by a 10 -min warm-up, wherein the participants performed three to five repetitions with increasing loads (i.e., from $40 \%$ to $80 \%$ of the $1-\mathrm{RM}$ ).

\section{Dietary records}

To assess the adequacy of nutrient intake, a consecutive dietary record over 7 days was completed. Participants were asked to continue their usual dietary habits during the period of dietary recording and to be as accurate as possible in recording the amounts and types of food and fluid consumed. Each individual's diet was calculated using the Bilnut 4 software package (SCDA Nutrisoft, Cerelles, France) and the food composition tables published by the Tunisian National Institute of Statistics in 1978. Estimated nutrient intakes were referred to reference dietary intakes for physically active people $(5,30)$. The daily nutrient data showed that total calorie, macronutrient, and micronutrient intakes are situated in the interval of the reference dietary intakes for healthy Tunisian adults.

\section{Blood sampling and analysis}

Fasting blood samples were collected from a forearm vein at rest, $3 \mathrm{~min}$ and $48 \mathrm{~h}$ after each training session. At rest and after $48 \mathrm{~h}$, blood samples were collected after $5 \mathrm{~min}$ of being seated. Samples were placed in an ice bath and centrifuged immediately at $2,500 \mathrm{r} / \mathrm{min}(\times \mathrm{g})$ and $4{ }^{\circ} \mathrm{C}$ for $10 \mathrm{~min}$. Aliquots of the resulting plasma were stored at $-80{ }^{\circ} \mathrm{C}$ until analyzed.

All samples were analyzed in the same assay run to eliminate inter-assay variance. All assays were performed in duplicate in the same laboratory with the simultaneous use of a control serum from Randox. WBC was generally performed within $3 \mathrm{~h}$ (using total blood) in a multichannel automated blood cell analyzer Beckman Coulter Gen system-2 (Coulter T540). CK, LDH, UA, TBIL, and C-reactive protein (CRP) were determined spectrophotometrically using Architect Ci 4100 d'ABOTT, respectively, by N-acetyl-L-cysteine, oxidation of lactate on pyruvate, uricase, diazonium, and immunoturbidimetric methods. The intra-assay coefficient of variation for these parameters were $1.3 \%, 0.2 \%, 0.5 \%, 0.5 \%$, and $1.16 \%$, respectively. Catalase (CAT) activity was measured following the decrease in $\mathrm{H}_{2} \mathrm{O}_{2}$ concentration. To check the activity, a kinetic curve had to be measured during $30 \mathrm{~s}$ at $\lambda=240 \mathrm{~nm}$ using a molar extinction coefficient $43.6 \mathrm{~cm}^{-1} \mathrm{M}^{-1}$ (43) to know the amount of $\mathrm{H}_{2} \mathrm{O}_{2}$ eliminated. To determine glutathione peroxidase (GPX) activity, the continuous decrease in NADPH concentration was measured, while GSH levels were maintained, following the Flohe and Gunzler (14) methods. The molar extinction coefficient used for the calculations is $\varepsilon=6.22 \times$ $10^{3} \mathrm{~cm}^{-1} \mathrm{M}^{-1}$. MDA was measured as reported by Wong et al. (44). Plasma proteins are precipitated with methanol and removed from the reaction mixture by centrifugation. The protein-free extract is fractionated by HPLC on a column of octadecyl silica gel to separate the MDA-thiobarbituric acid (TBA) adduct from interfering chromogens. The MDA-TBA 
adduct is eluted from the column with methanol/phosphate buffer and quantified spectrophotometrically at $\lambda=532 \mathrm{~nm}$. Plasma lipoperoxide concentrations are computed by reference to a calibration curve prepared by assays of tetraethoxypropane.

\section{Statistical analysis}

All statistical tests were processed using STATISTICA 10.0 Software (Stat-Soft, MaisonAlfort, France). Following normality confirmation using the Shapiro-Wilks $W$-test, paired simple $t$-test was applied to analyze the effect of each training session (i.e., morning, afternoon, and evening) on the biochemical parameters. To analyze the diurnal variation of the same parameters at rest, immediately and $48 \mathrm{~h}$ after the training sessions, one-way ANOVA [three levels (TOD)] was used for each period. Post hoc least significant difference (LSD) tests were conducted when significant main effects were found. Effect sizes were calculated as partial eta-squared $\left(\eta_{p}{ }^{2}\right)$ for the ANOVA analysis and as Cohen's $d$ for the paired sample $t$-test to assess the practical significance of the findings. Pearson correlation was used to assess the relationship between the studied parameters. Significance was set at $p<0.05$.

\section{Results}

The immediate biochemical responses (i.e., $3 \mathrm{~min}$ ) to the different training sessions were shown in Tables I-III. As these tables indicate, significant increases in the rate of lipid peroxidation and the antioxidant activities were shown after the morning (Table I), afternoon (Table II), and evening (Table III) training sessions compared to the pre-training values ( $p<$ 0.001 for MDA, UA, CAT, and GPX and $p<0.01, p<0.05$, and $p<0.01$ for TBIL at 08:00, 14:00, and 18:00, respectively). Concerning markers of muscle damage, significant increases were shown for the CK only after the morning and evening training sessions $(p<0.05)$ and for LDH after the evening training session $(p<0.01)$. For CRP, a significant increase was shown after the morning session only $(p<0.01)$. However, for WBC and core temperature, no significant changes were recorded after the different training sessions. Significant correlations were shown between $\mathrm{CK}$ and MDA and CK and UA in the morning and evening ( $p<0.01, r=0.6$ for CK-MDA and $p<0.01, r=0.66$ and $p<0.001, r=0.82$ for CK-UA), between CK and GPX in the morning and afternoon sessions ( $p<0.05, r=0.54$ and $p<0.01, r=0.6$, respectively), between CK and CAT in the evening training session $(p<0.001, r=0.73)$, and between $\mathrm{CK}$ and WBC in the morning training session $(p<0.05$, $r=-0.5)$. Regarding the inflammatory markers, CRP was highly correlated $(p<0.001)$ with WBC in the different training sessions $(r=0.76, r=0.77$, and $r=0.83$, respectively, at 08:00, 14:00, and 18:00). Similarly, WBC was correlated $(p<0.05)$ to core temperature during the morning and evening sessions $(r=0.6$ and $r=-0.5$, respectively).

Table IV shows the rest values of the biological markers recorded at 08:00, 14:00, and 16:00. A significant diurnal variation of core temperature, markers of muscle damage, and the majority of antioxidant parameters was observed with: (1) higher values recorded in the evening compared to the morning for temperature, WBC, CK, LDH, UA, CAT, and GPX ( $p<0.05, p<0.01, p<0.01, p<0.001, p<0.05, p<0.001$, and $p<0.001$, respectively); (2) there were higher values in the afternoon compared to the morning for temperature, LDH, UA, CAT, and GPX ( $p<0.05, p<0.001, p<0.05, p<0.01$, and $p<0.001$, respectively); and (3) higher values in the evening compared to the afternoon for the WBC $(p<0.01)$. In this table, CK was correlated $(p<0.05)$ to MDA, UA, GPX $(r=0.53, r=0.52$, 
Ammar et al.

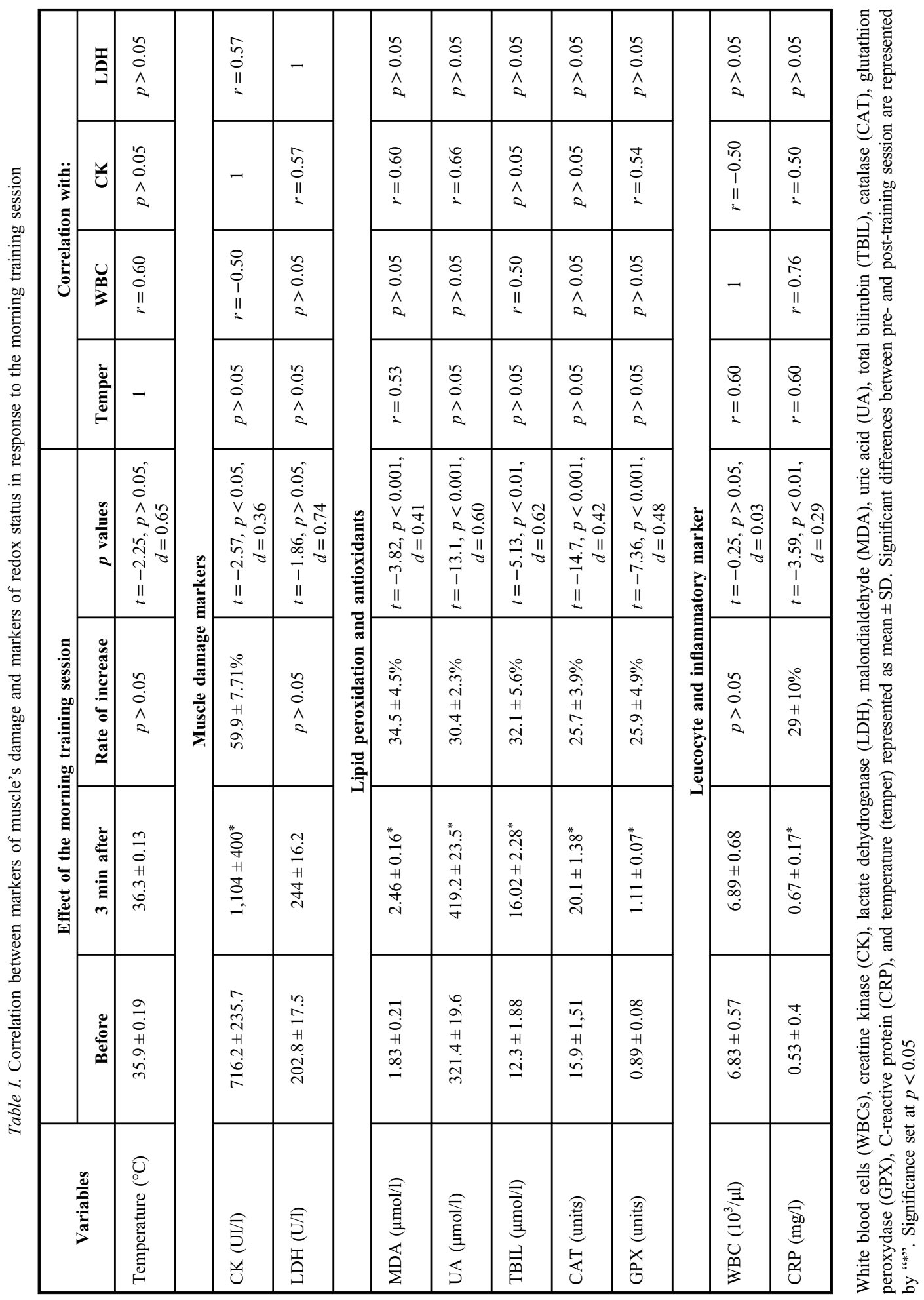




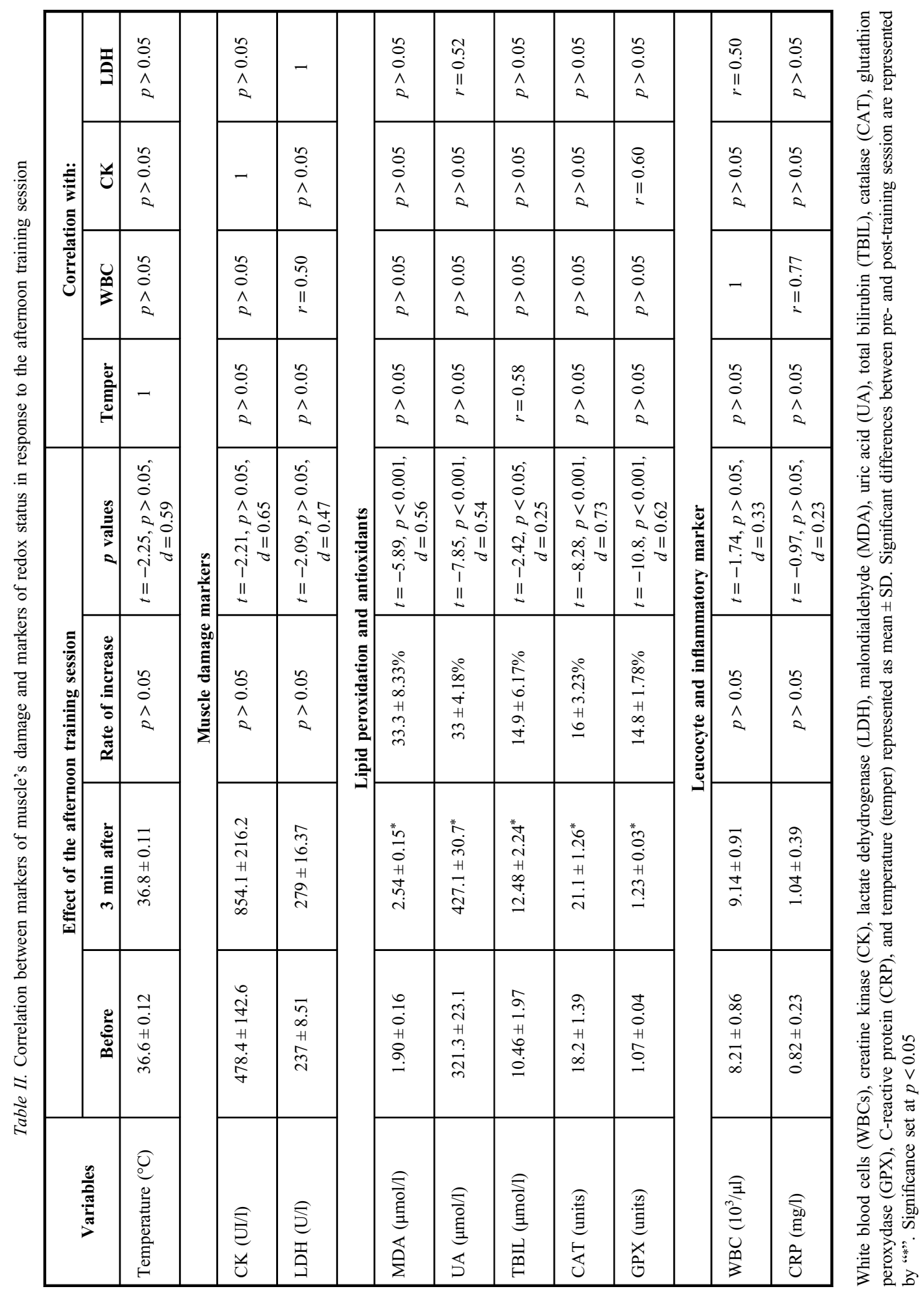


Ammar et al.

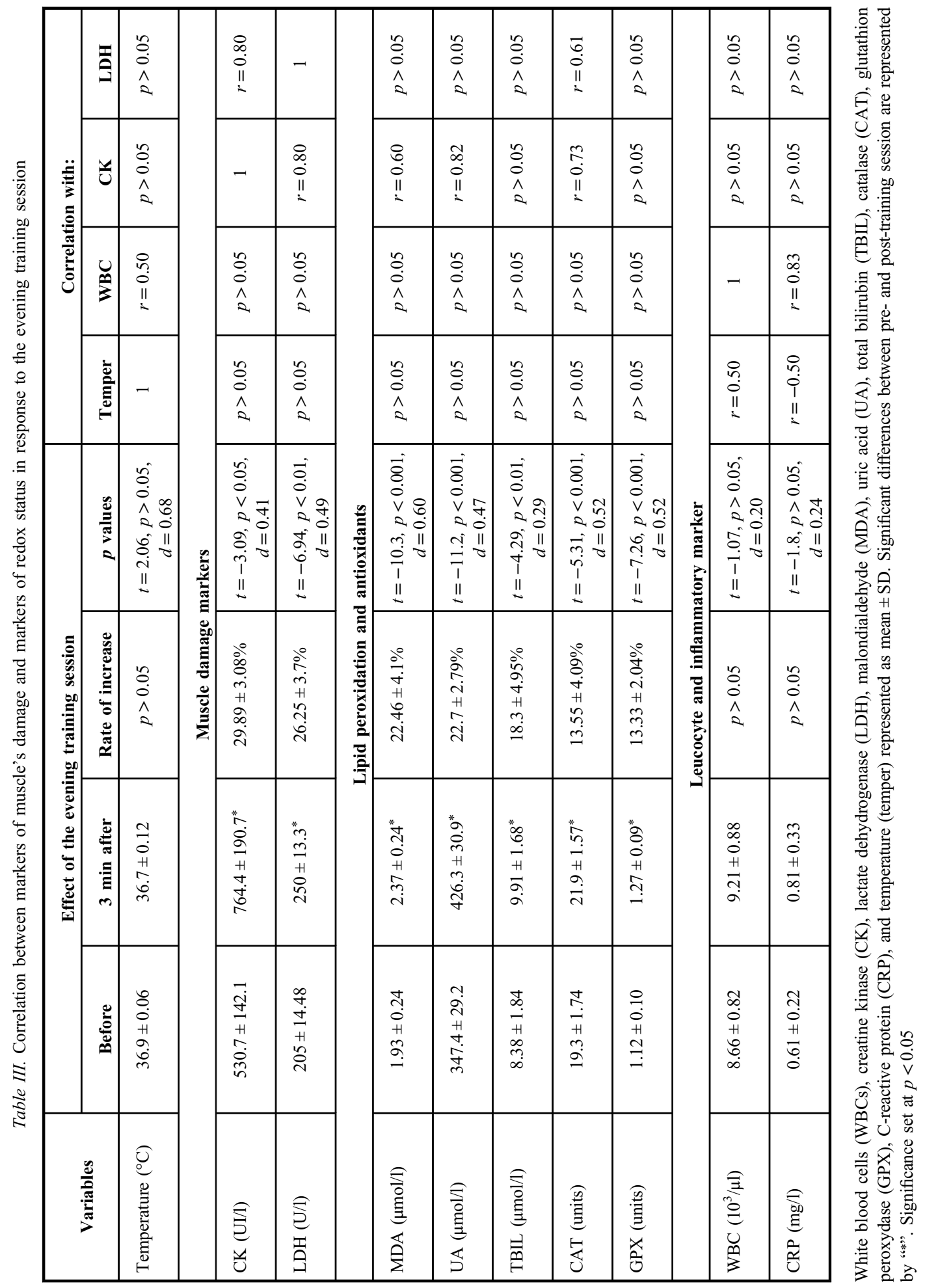


TOD effect on biological reponses to weightlifting training

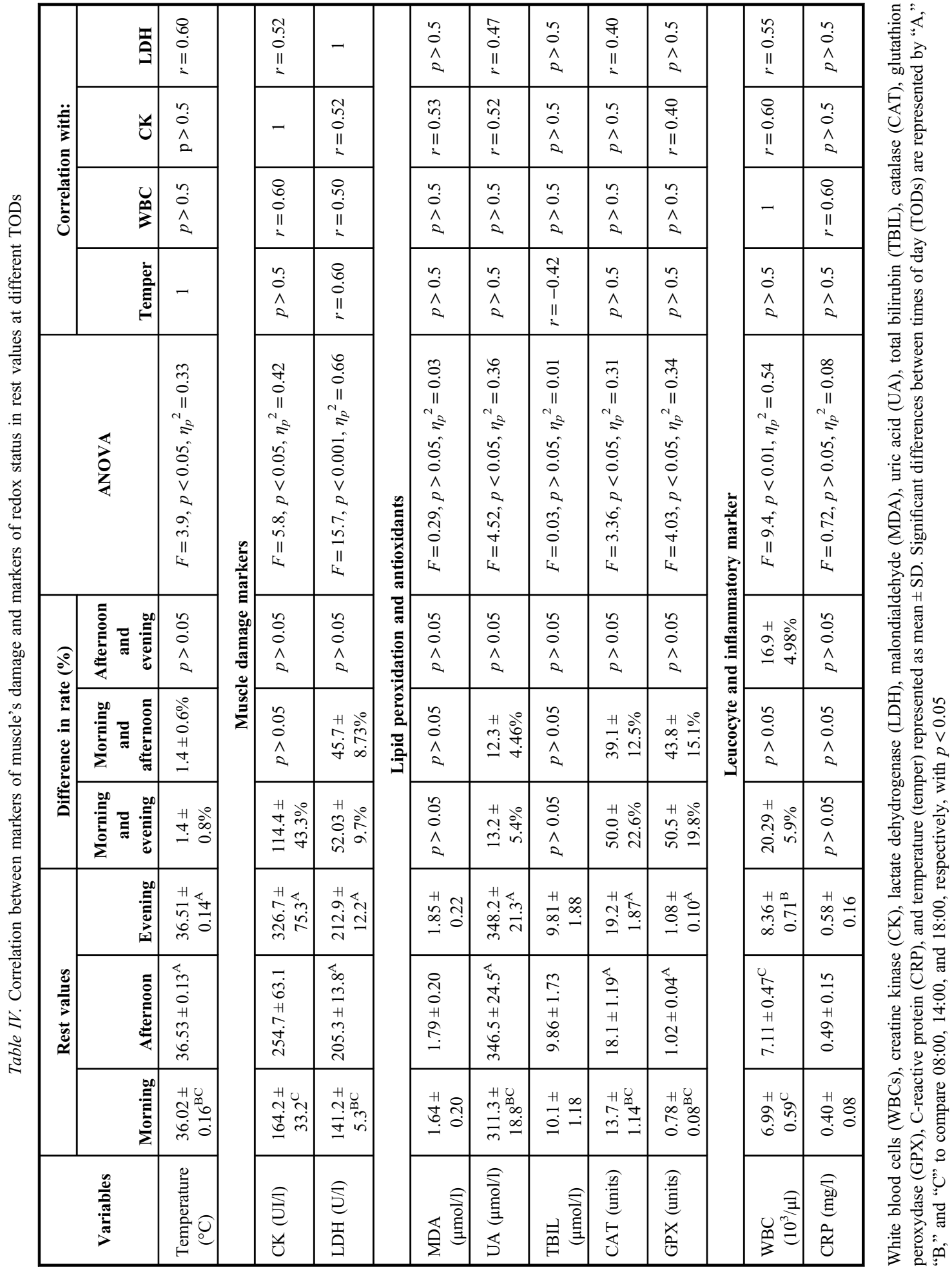


and $r=0.4$, respectively) and to $\mathrm{WBC}(p<0.01, r=0.6)$. LDH was correlated to $\mathrm{WBC}$, $\mathrm{UA}$, and CAT $(p<0.01, r=0.55$ for WBC; $p<0.05, r=0.47$ and $r=0.4$, respectively, for $\mathrm{UA}$ and CAT). CRP was correlated to $\mathrm{WBC}(p<0.01, r=0.6)$ and core temperature was correlated $(p<0.05)$ to LDH and TBIL ( $r=0.60$ and $r=-0.42$, respectively).

The diurnal variations of the different parameters studied (i.e., temperature, markers of muscle damage, and markers of redox status and CRP) immediately and $48 \mathrm{~h}$ after the training sessions were shown in Tables V and VI, respectively. Immediately after the training sessions (Table V), the results showed a significant TOD effect on core temperature, WBC, and TBIL values $\left(F=6.7, p<0.01, \eta_{p}{ }^{2}=0.45, F=7.1, p<0.01, \eta_{p}{ }^{2}=0.47\right.$, and $F=6.1$, $\left.p<0.05, \eta_{p}{ }^{2}=0.43\right)$ with higher values registered in the evening and afternoon compared to the morning for core temperature and $\operatorname{WBC}(p<0.05$ and $p<0.01$, respectively). However, lower values were registered immediately after the evening compared to the morning for TBIL $(p<0.01)$. As indicated in this table, significant correlations between CK and UA $(p<0.05, r=0.42)$, between CRP and WBC $(p<0.001, r=0.7)$, and between CRP and LDH $(p<0.05, r=0.43)$ were shown. After $48 \mathrm{~h}$ of recovery (Table VI), the results showed a significant diurnal variation only for core temperature and WBC with higher values recorded in the evening and afternoon compared to the morning $(p<0.001)$. However, the diurnal variation was removed for the remaining parameters. Significant correlations were observed between CK and UA $(p<0.05, r=0.53)$, between CRP and WBC $(p<0.001, r=0.73)$, between WBC and core temperature $(p<0.05, r=0.46)$, and between WBC and GPX $(p<0.05, r=0.4)$.

\section{Discussion}

The main findings of the present study were: (1) no significant changes for core temperature and WBC after the training sessions at the different TOD was found; (2) redox markers (i.e., MDA, UA, TBIL, CAT, and GPX) increased from resting values to post-training with a higher rate of increases registered in the morning; and (3) significant post-training increases were recorded for markers of muscle damage in the morning and evening excepting CRP only at 08:00. Moreover, in response to the weightlifting training session, significant correlations were reported during the: (1) morning and evening between CK and MDA levels and (2) morning between CRP and MDA and CK and WBC. In resting state, a significant diurnal variation in core temperature, WBC, $\mathrm{CK}, \mathrm{LDH}, \mathrm{UA}, \mathrm{CAT}$, and GPX was registered with higher values at 18:00 compared to 08:00. Significant correlations were observed between: (1) CK and WBC, MDA, UA, and GPX and (2) between LDH and core temperature, WBC, $\mathrm{UA}$, and CAT. Immediately and $48 \mathrm{~h}$ after training sessions, the diurnal variations persist only for core temperature and WBC and significant correlations were observed only between $\mathrm{CK}$ and $\mathrm{UA}$ and $\mathrm{WBC}$ and CRP.

\section{Relationship between redox status and markers of muscle damage during weightlifting training sessions}

The present results showed no significant differences between pre- and post-training for core temperature and WBC at the different TODs. However, markers of muscle damage increased after the training sessions at 08:00 and 18:00 with a higher rate of increase in the morning for CK $(59.9 \pm 7.71 \%$ versus $29.89 \pm 3.08 \%)$. These findings are partially in line with the previous studies of Yalcin et al. (45) and Hammouda et al. $(16,17)$ who showed a significant 
TOD effect on biological reponses to weightlifting training

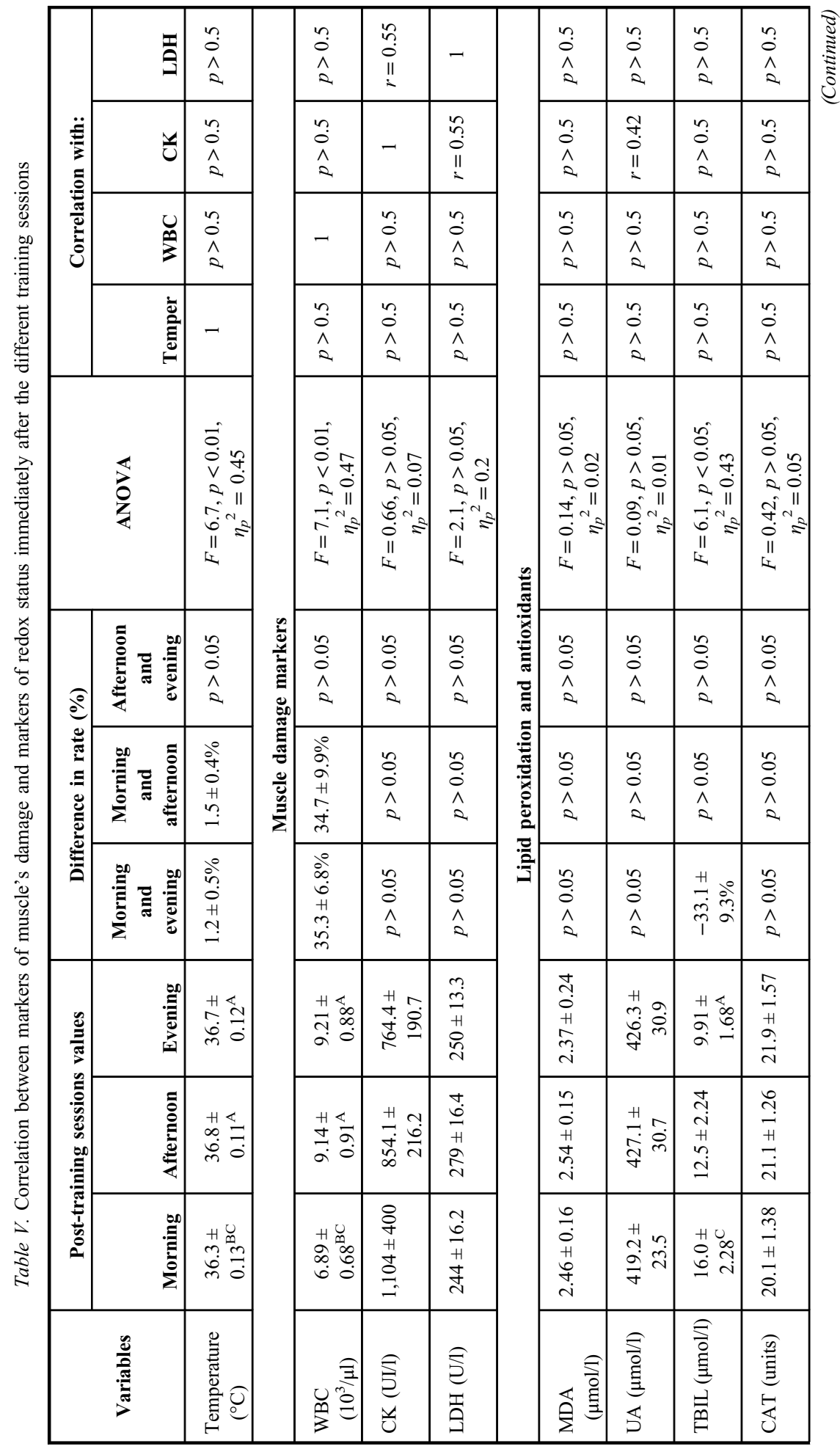




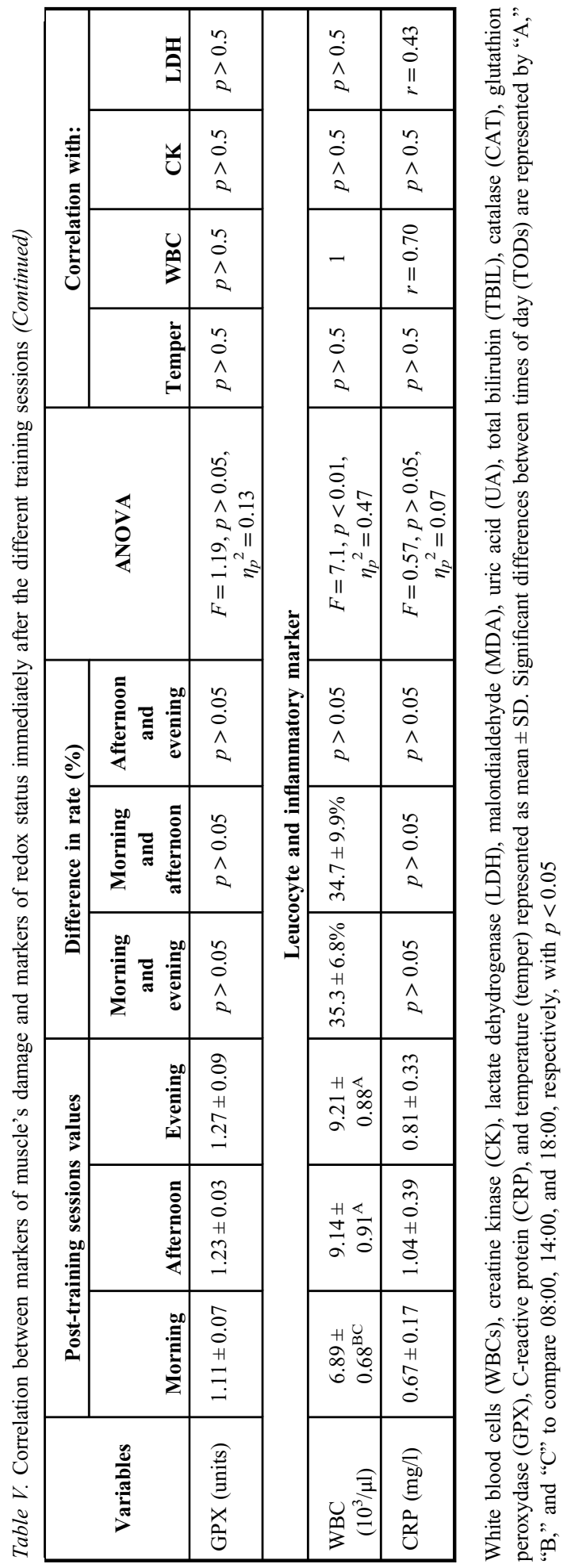


TOD effect on biological reponses to weightlifting training

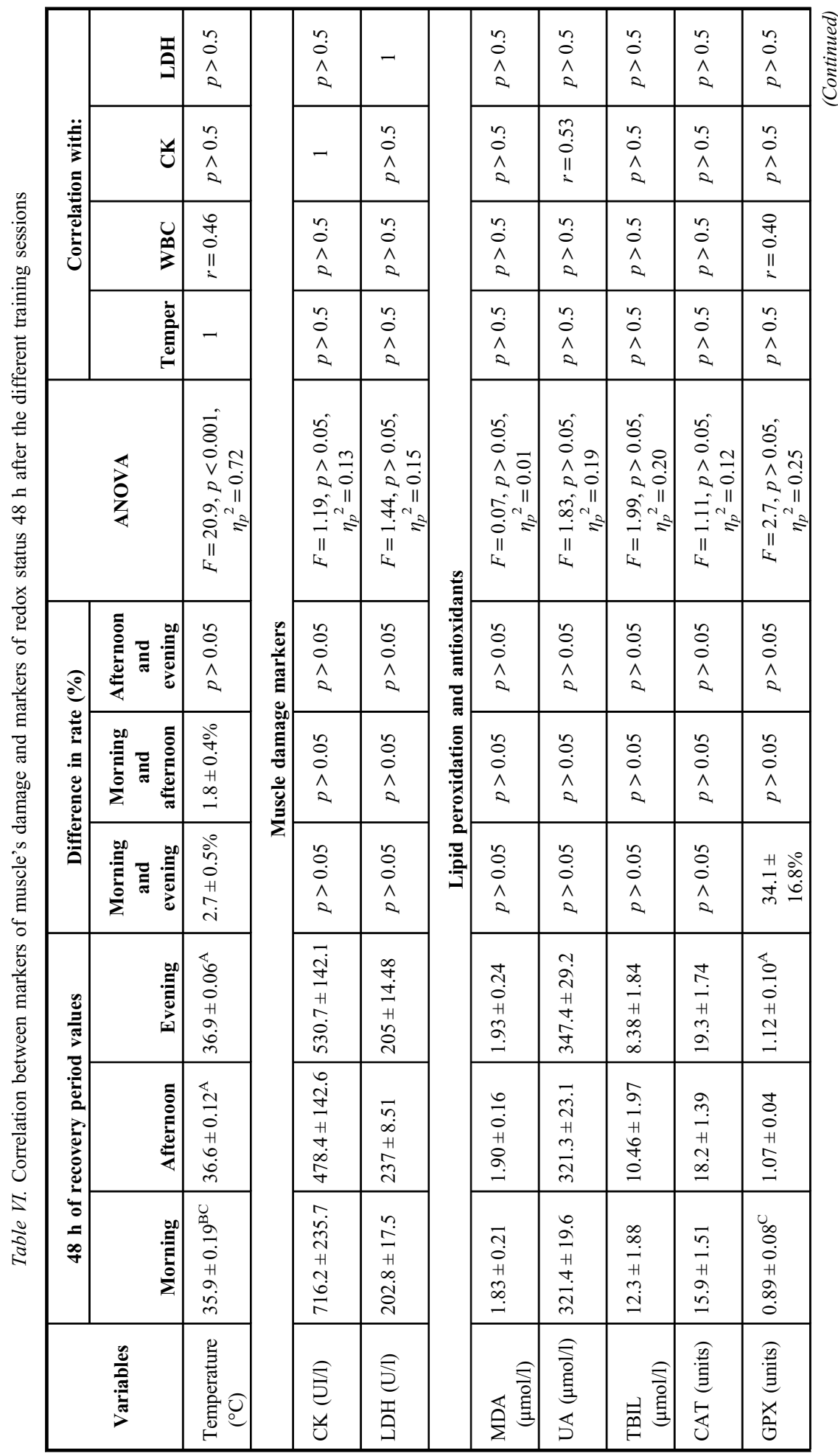




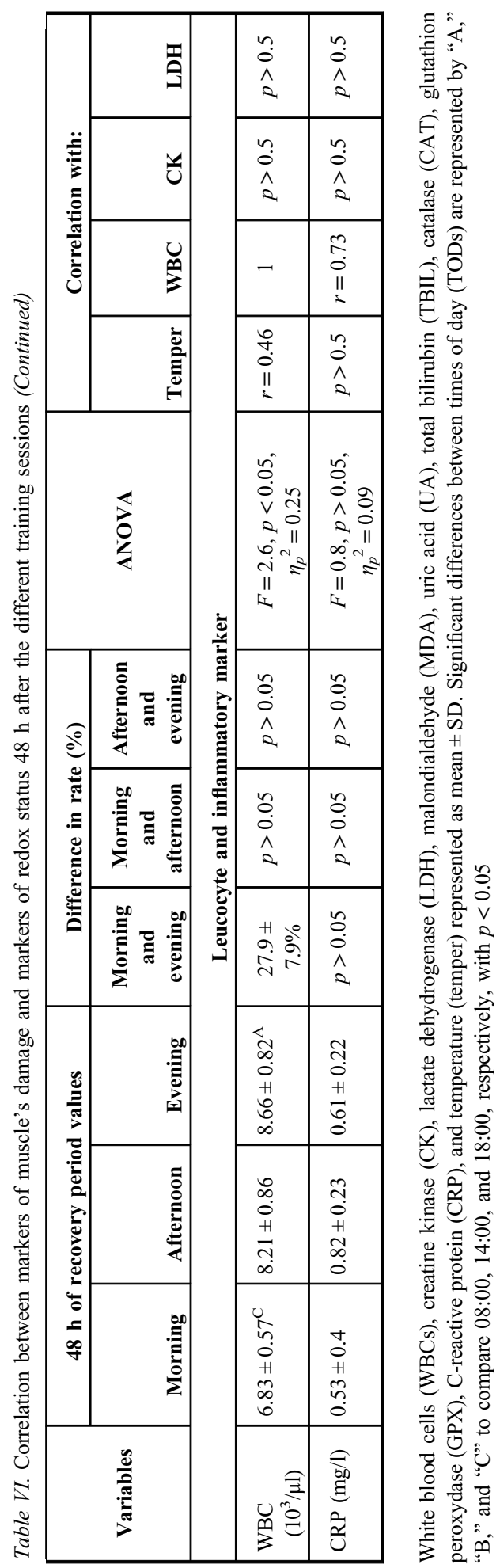


increase in $\mathrm{CK}, \mathrm{LDH}$, and WBC whatever the TOD of physical exercises has been. The present study showed significant pre-post training increases in MDA, UA, TBIL, CAT, and GPX at different TODs with a higher rate of increase observed in the morning compared to the afternoon and the evening values (e.g., MDA: morning $=34.5 \pm 11.9 \%$ versus evening $=$ $22.46 \pm 4.1 \%)$. In agreement, the previous studies $(4,16,21)$ reported the increases in lipid peroxidation (i.e., MDA) and antioxidant defenses (i.e., CAT, UA, and TBIL) after intense physical exercise. The present result showed a significant correlation between CK and MDA in the morning and evening. These findings confirm those of Liu et al. (25) and Akbulut et al. (1) after a resistance training session. Likewise, in line with the result of Nakajima et al. (28), the present study indicates a significant difference pre- to post-training in CRP only in the morning. The significant increase of CRP, CK, and MDA at this TOD and the significant correlation between CRP-WBC, CRP-CK, and CK-MDA, confirmed that inflammatory responses could be due to generalized muscle damage induced by a high level of oxidative stress (42).

\section{Relationship between redox status and markers of muscle damage at rest and after training sessions}

The present results showed that resting oral temperature, $\mathrm{WBC}, \mathrm{CK}$, and $\mathrm{LDH}$ were higher at 18:00 than 08:00. This confirms the results of the previous studies in which the authors found that the resting levels of WBC and biochemical markers of muscle damage were higher in the evening close to the acrophase of oral temperature $(16-18,39)$. These results confirmed the suggestion that circadian changes in core temperature would increase the activity of enzymes, such as phosphofructokinase and LDH (12). In this context, significant correlations between resting core temperature and $\mathrm{LDH}$ and between $\mathrm{WBC}$ and $\mathrm{CK}$ and $\mathrm{LDH}$ were registered. These findings confirmed that the presence of leucocytosis at a specific TOD could be due to the high concentrations of catecholamines (23) and to the general increase in muscle damage (34). The present study showed higher antioxidant activity in the evening and no diurnal variation for MDA. However, in healthy sedentary subjects, the previous studies $(12,15-20,22,23,38,45)$ showed a higher efficiency of the antioxidant system in the early morning (i.e., peak of CAT, UA, and TBIL recorded at 06:00) and a greater rate of lipid peroxidation in the early evening (i.e., peak of MDA occurred at 18:00). These divergences between elite weightlifter and healthy sedentary subjects could be due to the training level of the subjects and the effect of training at a specific TOD (i.e., participants of the present study regularly trained between 15:30 and 17:30). In this context, Bloomer et al. (7, 8), Subudhi et al. (40), and Ammar et al. (2) indicated that being experienced resistance trained could result in a lower MDA and muscle damage levels. On the other hand, the previous studies suggest a link between the diurnal variation of the oxidative stress and the muscle damage markers $(11,32)$. Cudney et al. (11) suggest that lipid peroxidation could be in the origin of hematological and muscle damage circadian rhythm and could even alter the whole circadian system. Petibois et al. (32) suggest that the increases in TBIL and UA are thought to reflect the imbalances in protein metabolic homeostasis that increased protein catabolism associated with tissue damage (i.e., increase in $\mathrm{CK}$ and LDH levels). The present results confirm the majorities of these suggestions and showed that resting: (1) CK was significantly correlated to MDA, UA, and GPX and (2) LDH was correlated to UA and CAT. Furthermore, given the similarity in the time of peak between core temperature and MDA (20), Kanabrocki et al. (22) suggest a correlation between increased oxidative damage and increased rate of anabolic-catabolic events (e.g., oral temperature). The present results are in disagreement 
with this suggestion. Indeed, at resting state, core temperature was only negatively correlated to TBIL.

Immediately and $48 \mathrm{~h}$ after the training sessions, the present study showed that the diurnal variation persisted only for core temperature and WBC which is in line with the previous findings after the repeated sprint and Wingate exercise $(16,17)$. However, the present findings showed the suppression of TOD effect in markers of muscle damage and antioxidant activities immediately and $48 \mathrm{~h}$ after weightlifting training sessions. These results suggest that the effect of intensive weightlifting exercises dominates the TOD effect in marker of muscle damage and antioxidant activity. However, for core temperature and WBC, the TOD effect dominates the physical exercise's effect.

Concerning the relationship between redox status and markers of muscle damage, the present results showed that the majorities of resting significant correlations were suppressed immediately and $48 \mathrm{~h}$ after the resistance training session. Therefore, the authors suggest the existence of further underlying mechanisms [i.e., such as catecholamine Kanabrocki et al. (23)] which can be in the origin of muscle damage response to high-intensity weightlifting exercises. Concerning the CRP, our results showed the absence of diurnal variations at resting state and immediately and $48 \mathrm{~h}$ after the training sessions. These findings are in line with those of Miles et al. (27) who investigated the diurnal variation of CRP during eccentric exercises and showed the absence of significant daily fluctuation at both pre- and postphysical exercises. Moreover, the present study shows that CRP and WBC are correlated under different conditions (i.e., resting state, immediately and $48 \mathrm{~h}$ after the training sessions) and this suggests that WBC could also be a marker of inflammation during physical exercise.

\section{Conclusion}

In conclusion, the present study suggests a higher sensitivity to the resistance exercises for the lipid peroxidation and antioxidant activity compared to WBC and markers of muscle damage. However, core temperature and WBC were more sensible to TOD than the remaining parameters (i.e., lipid peroxidation, antioxidant activity, and markers of muscle damage and CRP). Indeed, immediately and $48 \mathrm{~h}$ after the training sessions, the present study showed that the diurnal variation already observed in resting state persist only for core temperature and WBC. This finding could be explained, as reported in the present study, by the fact that the resting circadian rhythm of enzyme activities (e.g., $\mathrm{LDH}$ ) and $\mathrm{CK}$ and the diurnal fluctuation in core temperature, lipid peroxidation, and activities of some potent antioxidants are causally linked. However, the suppression of this relationship after the intense resistance exercises suggests the participation of further underlying mechanisms in the muscle damage process that needs further studies.

\section{Practical Applications}

Given the fact that the increase in the inflammatory markers and the higher rate of increase pre-post training session for the redox and the muscle damage markers were registered during the morning, this session seems to generate higher muscle damage, inflammatory, and oxidative responses. Therefore, weightlifting coaches should avoid scheduling their training sessions in the morning hours. 


\section{Limits}

The majority of the present study subjects are used to participate essentially in afternoon/ evening training schedules which could induce a temporal specificity adaptation upon biological measures. Therefore, further studies could examine the same protocol with subjects habitually trained in the morning hours.

\section{Acknowledgement}

The authors would like to thank all the participants for their maximal effort and cooperation.

\section{Conflict of interest}

The authors report no conflict of interest. The authors are solely responsible for the content and writing of this paper.

\section{REFERENCES}

1. Akbulut H, Akbulut KG, Icli F, Büyükcelik A: Daily variations of plasma malondialdehyde levels in patients with early breast cancer. Cancer Detect. Prev. 27, 122-126 (2003)

2. Ammar A, Chtourou H, Hammouda O, Trabelsi K, Chiboub J, Turki M, AbdelKarim O, El Abed K, Ben Ali M, Hoekelmann A, Souissi N: Acute and delayed responses of C-reactive protein, malondialdehyde and antioxidant markers after resistance training session in elite weightlifters: effect of time of day. Chronobiol. Int. 32, 1211-1222 (2015)

3. Ammar A, Chtourou H, Trabelsi K, Padulo J, Turki M, El Abed K, Hoekelmann A, Hakim A: Temporal specificity of training: intra-day effects on biochemical responses and Olympic-Weightlifting performances. J. Sports Sci. 33, 358-368 (2015)

4. Antoncic-Svetina M, Sentija D, Cipak A, Milicic D, Meinitzer A, Tatzber F, Andrisic L, Zelzer S, Zarkovic N: Ergometry induces systemic oxidative stress in healthy human subjects. Tohoku J. Exp. Med. 221, 43-48 (2010)

5. Aounallah-Skhiri H, Traissac P, El Ati J, Eymard-Duvernay S, Landais E, Achour N, Delpeuch F, Ben Romdhane H, Maire B: Nutrition transition among adolescents of a south-Mediterranean country: dietary patterns, association with socio-economic factors, overweight and blood pressure. A cross-sectional study in Tunisia. Nutr. J. 10, 38-55 (2011)

6. Bird SP, Tarpenning KM, Marino FE: Designing resistance training programmes to enhance muscular fitness. A review of the acute programme variables. Sports Med. 35, 841-851 (2005)

7. Bloomer RJ, Falvo MJ, Fry AC, Schilling BK, Smith WA, Moore CA: Oxidative stress response in trained men following repeated squats or sprints. Med. Sci. Sports Exerc. 38, 1436-1442 (2007)

8. Bloomer RJ, Goldfarb AH, Wideman L, McKenzie MJ, Consitt LA: Effects of acute aerobic and anaerobic exercise on blood markers of oxidative stress. J. Strength Cond. Res. 19, 276-285 (2005)

9. Bougard C, Moussay S, Gauthier A, Espié S, Davenne D: Effects of waking time and breakfast intake prior to evaluation of psychomotor performance in the early morning. Chronobiol. Int. 26, 324-336 (2009)

10. Brancaccio P, Giuseppe L, Nicola M: Biochemical markers of muscular damage. Clin. Chem. Lab. Med. 48, 757-767 (2010)

11. Cudney LE, Sassi RB, Behr GA, Streiner DL, Minuzzi L, Moreira JC, Frey BN: Alterations in circadian rhythms are associated with increased lipid peroxidation in females with bipolar disorder. Int. J. Neuropsychopharmacol. 17, 715-722 (2014)

12. Dalton B, McNaughton L, Davoren B: Circadian rhythms have no effect on cycling performance. Int. J. Sports Med. 18, 538-542 (1997)

13. Dosseville F, Laborde S, Lericollais R: Validation of a chronotype questionnaire including an amplitude dimension. Chronobiol. Int. 30, 639-648 (2013)

14. Flohe L, Gunzler WA: Assays of glutathione peroxidase. Methods Enzymol. 105, 114-121 (1984)

15. Gutenbrunner C: Circadian variations of the serum creatine kinase level: a masking effect? Chronobiol. Int. 17, 583-590 (2000) 
16. Hammouda O, Chtourou H, Chahed H, Ferchichi S, Chaouachi A, Kallel C, Miled A, Chamari K, Souissi N: High intensity exercise affects diurnal variation of some biological markers in trained subjects. Int. J. Sports Med. 33, 886-891 (2012)

17. Hammouda O, Chtourou H, Chahed H, Ferchichi S, Kallel C, Miled A, Chamari K, Souissi N: Diurnal variations of plasma homocysteine, total antioxidant status, and biological markers of muscle injury during repeated sprint: effect on performance and muscle fatigue - a pilot study. Chronobiol. Int. 28, 958-967 (2011)

18. Hammouda O, Chtourou H, Chaouachi A, Chahed H, Bellimem H, Chamari K, Souissi N: Time-of-day effects on biochemical responses to soccer-specific endurance in elite Tunisian football players. J. Sports Sci. 31, 963-971 (2013)

19. Haus E, Lakatua D, Jacqueline S, Sackett-Lundeen L: Chronobiology in hematology and immunology. Am. J. Anat. 168, 467-517 (1983)

20. Haus E, Lakauta DJ, Sackett-Lundeen L, Swoyer J (1984): Chronobiology in laboratory medicine. In: Clinical Aspects of Chronobiology, ed Rietveld WJ, Hoechst Pharma, Amsterdam, pp. 13-83

21. Inal M, Akyüz F, Turgut A, Getsfrid WM: Effect of aerobic and anaerobic metabolism on free radical generation swimmers. Med. Sci. Sports Exerc. 33, 564-567 (2001)

22. Kanabrocki EL, Hermida RC, Scott GS, Bremner WF, Ryan MD, Ayala DE, Third JL, Shirazi P, Nemchausky BA, Hooper DC: Circadian variation in oxidative stress markers in healthy and type II diabetic. Chronobiol. Int. 19, 423-439 (2002)

23. Kanabrocki EL, Sothern RB, Scheving LE, Vesely DL, Tsai TH, Shelstad J, Cournoyer C, Greco J, Mermall H, Ferlin H, Nemchauskya BM, Bushnella DL, Kaplana E, Kahnf S, Augustinef G, Holmesf E, Rumbyrtf J, Sturtevantf RP, Sturtevantf F, Bremnerf F, Thirdf JLHC, McCormickg JB, Dawsong S, Sackett-Lundeenh L, Hausch E, Halbergc F, Paulyd JE, Olwind JH: Reference values for circadian rhythms of 98 variables in clinically healthy men in fifth decade of life. Chronobiol. Int. 7, 445-461 (1990)

24. Kraemer WJ, Fry AC (1995): Strength testing: development and evaluation of methodology. In: Physiological Assessment of Human Fitness, eds Maud P, Foster C, Human Kinetics, Champaign, IL, pp. 115-138

25. Liu JF, Chang WY, Chan KH, Tsai WY, Lin CL, Hsu MC: Blood lipid peroxides and muscle damage increased following intensive resistance training of female weightlifters. Ann. N. Y. Acad. Sci. 1042, 255-261 (2005)

26. Meltzer DE: Age dependence of Olympic Weightlifting ability. Med. Sci. Sports Exerc. 26, 1053-1067 (1994)

27. Miles MP, Andring JM, Pearson SD, Gordon LK, Kasper C, Depner CM, Kidd JR: Diurnal variation, response to eccentric exercise, and association of inflammatory mediators with muscle damage variables. J. Appl. Physiol. $104,451-458$ (2008)

28. Nakajima T, Kurano M, Hasegawa T, Takano H, Iida H, Yasuda T, Fukuda T, Madarame H, Uno K, Meguro K, Shiga T, Sagara M, Nagata T, Maemura K, Hirata Y, Yamasoba T, Nagai R: Pentraxin 3 and high-sensitive C-reactive protein are independent inflammatory markers released during high-intensity exercise. Eur. J. Appl. Physiol. 110, 905-913 (2010)

29. Nosaka K, Clarkson PM: Muscle damage following repeated bouts of high force eccentric exercise. Med. Sci. Sports Exerc. 27, 1263-1269 (1995)

30. Otten JJ, Hellwig JP, Meyers LD (2006): Dietary Reference Intakes: The Essential Guide to Nutrient Requirements. National Academy Press, Washington, DC

31. Padulo J, Laffaye G, Chaouachi A, Chamari K: Bench press exercise: the key points. J. Sports Med. Phys. Fitness 55, 604-608 (2015)

32. Petibois C, Cazorla G, Déléris G, Gin H: Clinical diagnosis of overtraining using blood tests: current knowledge. Rev. Med. Interne 22, 723-736 (2001)

33. Pettersson J, Hindorf U, Persson P, Bengtsson T, Malmqvist U, Werkström V, Ekelund M: Muscular exercise can cause highly pathological liver function tests in healthy men. Br. J. Clin. Pharmacol. 65, 253259 (2008)

34. Pyne DB: Exercise-induced muscle damage and inflammation: a review. Aust. J. Sci. Med. Sport 26, 49-52 (1994)

35. Rietjens GJ, Kuipers H, Adam JJ, Saris WH, van Breda E, van Hamont D, Keizer HA: Physiological, biochemical and psychological markers of strenuous training-induced fatigue. Int. J. Sports Med. 26, 16-26 (2006)

36. Rivera-Coll A, Fuentes-Arderiu X, Diez-Noguera A: Circadian rhythms of serum concentration of 12 enzymes of clinical interest. Chronobiol. Int. 10, 190-200 (1993)

37. Sani M, Sebai H, Ghanem-Boughanmi N, Naceur A, Boughattas M, Ben-Attia M: Circadian (about 24-hour) variation in malondialdehyde content and catalase activity of mouse erythrocytes. Redox Rep. 20, 26-32 (2014) 
38. Singh R, Singh RK, Tripathi AK, Gupta N, Kumar A, Singh AK, Mahdi AA, Prasad R, Singh RK: Circadian periodicity of plasma lipid peroxides and anti-oxidant enzymes in pulmonary tuberculosis. Indian J. Clin. Biochem. 19, 14-20 (2004)

39. Souissi N, Bessot N, Chamari K, Gauthier A, Sesboue B, Davenne D: Effect of time of day on aerobic contribution to the 30-s Wingate test performance. Chronobiol. Int. 21, 739-748 (2007)

40. Subudhi AW, Davis SL, Kipp RW, Askew EW: Antioxidant status and oxidative stress in elite alpine ski racers. Int. J. Sport Nutr. Exerc. Metab. 11, 32-41 (2001)

41. Takahashi I, Umeda T, Mashiko T, Chinda D, Oyama T, Sugawara K, Nakaji S: Effects of rugby sevens matches on human neutrophil-related nonspecific immunity. Br. J. Sports Med. 41, 13-18 (2007)

42. Tidball JG: Mechanisms of muscle injury, repair, and regeneration. Compr. Physiol. 1, 2029-2062 (2011)

43. Wheeler CR, Salzman JA, Elsayed NM, Omaye ST, Korte DW: Automated assays for superoxide dismutase, catalase, glutathione peroxidase, and glutathione reductase activity. Anal. Biochem. 184, 193-199 (1990)

44. Wong SHY, Knight JA, Hopfer SM, Zaharia O, Leach CN Jr, Sunderman FW Jr: Lipoperoxides in plasma as measured by liquid-chromatographic separation of malondialdehyde-thiobarbituric acid adduct. Clin. Chem. 33, 214-220 (1987)

45. Yalcin O, Alpaslan E, Sedat M, Melek B, Oguzk B: Time course of hemorheological alterations after heavy anaerobic exercise in untrained human subjects. J. Appl. Physiol. 94, 997-1002 (2003) 